International Journal of Modern Physics D

(C) World Scientific Publishing Company

\title{
Composite Dark Matter and Puzzles of Dark Matter Searches
}

\author{
MAXIM YU. KHLOPOV \\ Virtual Institute of Astroparticle physics, APC laboratory \\ 10, rue Alice Domon et Léonie Duquet 75205 Paris Cedex 13, France, \\ Centre for Cosmoparticle Physics "Cosmion" and \\ National Research Nuclear University (Moscow Engineering Physics Institute) \\ 115409 Moscow, Russia \\ khlopov@apc.univ-paris7.fr \\ ANDREY G. MAYOROV \\ National Research Nuclear University (Moscow Engineering Physics Institute) \\ 115409 Moscow, Russia \\ mayorov.a.g@gmail.com \\ EVGENY YU. SOLDATOV \\ National Research Nuclear University (Moscow Engineering Physics Institute) \\ 115409 Moscow, Russia \\ Evgeny.Soldatov@cern.ch \\ Received Day Month Year \\ Revised Day Month Year \\ Communicated by Managing Editor
}

\begin{abstract}
Positive results of dark matter searches in DAMA/NaI and DAMA/LIBRA experiments, being put together with the results of other groups, can imply nontrivial particle physics solutions for cosmological dark matter. Stable particles with charge -2 , bound with primordial helium in O-helium "atoms" (OHe), represent a specific Warmer than Cold nuclear-interacting form of dark matter. Slowed down in the terrestrial matter, $\mathrm{OHe}$ is elusive for direct methods of underground Dark matter detection used in cryogenic experiments. However radiative capture of $\mathrm{OHe}$ by $\mathrm{Na}$ and I nuclei can lead to annual variations of energy release in the interval of energy $2-5 \mathrm{keV}$ in DAMA/NaI and DAMA/LIBRA experiments.
\end{abstract}

Keywords: Elementary particles, nuclear reactions, dark matter.

The widely shared belief is that the dark matter, corresponding to $25 \%$ of the total cosmological density, is nonbaryonic and consists of new stable particles. One can formulate the set of conditions under which new particles can be considered as candidates to dark matter (see e.g. Refs. 13 for review and reference): they should be stable, saturate the measured dark matter density and decouple from plasma and radiation at least before the beginning of matter dominated stage. The easiest way to satisfy these conditions is to involve neutral weakly interacting particles. However it is not the only particle physics solution for the dark matter 
problem. In the composite dark matter scenarios new stable particles can have electric charge, but escape experimental discovery, because they are hidden in atomlike states maintaining dark matter of the modern Universe.

It offers new solutions for the physical nature of the cosmological dark matter. The main problem for these solutions is to suppress the abundance of positively charged species bound with ordinary electrons, which behave as anomalous isotopes of hydrogen or helium. This problem is unresolvable, if the model predicts stable particles with charge -1 , as it is the case for tera-electrons 4|5]. To avoid anomalous isotopes overproduction, stable particles with charge -1 should be absent, so that stable negatively charged particles should have charge -2 only.

Elementary particle frames for heavy stable -2 charged species are provided by: (a) stable "antibaryons" $\bar{U} \bar{U}$ formed by anti- $U$ quark of fourth generation $617|8| 9$

(b) AC-leptons 910/11, predicted in the extension 10 of standard model, based on the approach of almost-commutative geometry 12 . (c) Technileptons and antitechnibaryons 13 in the framework of walking technicolor models (WTC) 14. (d) Finally, stable charged clusters $\bar{u}_{5} \bar{u}_{5} \bar{u}_{5}$ of (anti)quarks $\bar{u}_{5}$ of 5 th family can follow from the approach, unifying spins and charges 15 .

In the asymmetric case, corresponding to excess of -2 charge species, $X^{--}$, their positively charged antiparticles effectively annihilate in the early Universe. In all the models, in which new stable species belong to non-trivial representations of electroweak $\mathrm{SU}(2)$ group sphaleron transitions at high temperatures provide the relationship between baryon asymmetry and excess of -2 charge stable species.

After it is formed in the Standard Big Bang Nucleosynthesis (SBBN), ${ }^{4} \mathrm{He}$ screens the $\mathrm{X}^{--}$charged particles in $\left({ }^{4} \mathrm{He}^{++} \mathrm{X}^{--}\right)$O-helium "atoms" 7 .

In all the forms of O-helium, $X^{--}$behaves either as lepton or as specific "heavy quark cluster" with strongly suppressed hadronic interaction. Therefore O-helium interaction with matter is determined by nuclear interaction of $\mathrm{He}$. These neutral primordial nuclear interacting objects contribute to the modern dark matter density and play the role of a nontrivial form of strongly interacting dark matter 16 .

Here after a brief review of main features of OHe Universe we concentrate on its effects in underground detectors. We present a quantitative confirmation of the earlier guess $7|17| 18|19| 20$ that the positive results of dark matter searches in DAMA/NaI (see for review Ref. 21) and DAMA/LIBRA 22 experiments can be explained by effect of O-helium, resolving the controversy between these data and negative results of other experimental groups.

\section{O-helium Universe}

Following Refs. 7913 and 17 consider charge asymmetric case, when excess of $X^{--}$ provides effective suppression of positively charged species.

In the period $100 \mathrm{~s} \leq t \leq 300 \mathrm{~s}$ at $100 \mathrm{keV} \geq T \geq T_{o}=I_{o} / 27 \approx 60 \mathrm{keV},{ }^{4} H e$ has already been formed in the SBBN and virtually all free $X^{--}$are trapped by ${ }^{4} \mathrm{He}$ 
in O-helium "atoms" $\left({ }^{4} \mathrm{He}^{++} \mathrm{X}^{--}\right)$. Here the O-helium ionization potential is

$$
I_{o}=Z_{x}^{2} Z_{H e}^{2} \alpha^{2} m_{H e} / 2 \approx 1.6 \mathrm{MeV},
$$

where $\alpha$ is the fine structure constant, $Z_{H e}=2$ and $Z_{x}=2$ stands for the absolute value of electric charge of $X^{--}$. The size of these "atoms" is $7 / 11$

$$
R_{o} \sim 1 /\left(Z_{x} Z_{H e} \alpha m_{H e}\right) \approx 2 \cdot 10^{-13} \mathrm{~cm}
$$

Here and further, if not specified, we use the system of units $\hbar=c=k=1$.

Due to nuclear interactions with nuclei of cosmic plasma, the O-helium gas is in thermal equilibrium with plasma and radiation on the Radiation Dominance (RD) stage, while the energy and momentum transfer from plasma is effective. The radiation pressure acting on the plasma is then transferred to density fluctuations of the O-helium gas and transforms them in acoustic waves at scales up to the size of the horizon.

At temperature $T<T_{o d} \approx 200 S_{3}^{2 / 3} \mathrm{eV}$ the energy and momentum transfer from baryons to O-helium is not effective 713 because $n_{B}\langle\sigma v\rangle\left(m_{p} / m_{o}\right) t<1$, where $m_{o}$ is the mass of the OHe atom, $S_{3}=m_{o} /(1 \mathrm{TeV}), m_{p}$ is the mass of proton, $\sigma \approx \sigma_{o} \sim \pi R_{o}^{2} \approx 10^{-25} \mathrm{~cm}^{2}$ and $v=\sqrt{3 T / m_{p}}$ is the baryon thermal velocity. Then O-helium gas decouples from plasma. It starts to dominate in the Universe after $t \sim 10^{12} \mathrm{~s}$ at $T \leq T_{R M} \approx 1 \mathrm{eV}$ and O-helium "atoms" play the main dynamical role in the development of gravitational instability, triggering the large scale structure formation. The composite nature of O-helium determines the specifics of the corresponding dark matter scenario, which has qualitative feature of a Warmer Than Cold Dark Matter model 20.

Being decoupled from baryonic matter, the $\mathrm{OHe}$ gas does not follow the formation of baryonic astrophysical objects (stars, planets, molecular clouds...) and forms dark matter halos of galaxies. It can be easily seen that O-helium gas is collisionless for its number density, saturating galactic dark matter. Taking the average density of baryonic matter one can also find that the Galaxy as a whole is transparent for O-helium in spite of its nuclear interaction. Only individual baryonic objects like stars and planets are opaque for it.

O-helium atoms can be destroyed in astrophysical processes, giving rise to acceleration of free $X^{--}$in the Galaxy.

If the mechanisms of $X^{--}$acceleration are effective, the anomalous low $Z / A$ component of -2 charged $X^{--}$can be present in cosmic rays at the level 20/24 $X / p \sim n_{X} / n_{g} \sim 10^{-9} S_{3}^{-1}$, and be within the reach for PAMELA and AMS02 cosmic ray experiments.

In the framework of Walking Technicolor model the excess of both stable $X^{--}$ and $Y^{++}$is possible 18 , the latter being two-three orders of magnitude smaller, than the former. It leads to the two-component composite dark matter scenario with the dominant $\mathrm{OHe}$ accompanied by a subdominant WIMP-like component of

a The account for charge distribution in $H e$ nucleus leads to smaller value $I_{o} \approx 1.3 \mathrm{MeV} 23$. 
$\left(X^{--} Y^{++}\right)$bound systems. Technibaryons and technileptons can be metastable and decays of $X^{--}$and $Y^{++}$can provide explanation for anomalies, observed in high energy cosmic positron spectrum by PAMELA and in high energy electron spectrum by FERMI and ATIC.

O-helium collisions in the galactic bulge can lead to excitation of O-helium. If $2 \mathrm{~S}$ level is excited, pair production dominates over two-photon channel in the deexcitation by $E 0$ transition and positron production with the rate $3 \cdot 10^{42} S_{3}^{-2} \mathrm{~s}^{-1}$ is not accompanied by strong gamma signal. According to Ref. 25] this rate of positron production for $S_{3} \sim 1$ is sufficient to explain the excess in positron annihilation line from bulge, measured by INTEGRAL (see Ref. 26 for review and references). If $\mathrm{OHe}$ levels with nonzero orbital momentum are excited, gamma lines should be observed from transitions $(n>m) E_{n m}=1.598 \mathrm{MeV}\left(1 / m^{2}-1 / n^{2}\right)$ (or from the similar transitions corresponding to the case $I_{o}=1.287 \mathrm{MeV}$ ) at the level of $3 \cdot 10^{-4} S_{3}^{-2}\left(\mathrm{~cm}^{2} \mathrm{~s} \mathrm{MeVsr}\right)^{-1}$.

\section{O-helium in the terrestrial matter}

The evident consequence of the O-helium dark matter is its inevitable presence in the terrestrial matter, which appears opaque to O-helium and stores all its in-falling flux.

The nuclear cross section of the O-helium interaction with matter escapes the severe constraints on strongly interacting dark matter particles (SIMPs) $\frac{16}{16}$ imposed by the XQC experiment 27 . Therefore, a special strategy of direct O-helium search is needed, as it was proposed in Ref. 28 .

After they fall down terrestrial surface the in-falling $\mathrm{OHe}$ particles are effectively slowed down due to elastic collisions with matter. Then they drift, sinking down towards the center of the Earth with velocity

$$
V=\frac{g}{n \sigma v} \approx 80 S_{3} A_{m e d}^{1 / 2} \mathrm{~cm} / \mathrm{s} .
$$

Here $A_{\text {med }} \sim 30$ is the average atomic weight in terrestrial surface matter, $n=$ $2.4 \cdot 10^{24} / A_{\text {med }}$ is the number of terrestrial atomic nuclei, $\sigma v$ is the rate of nuclear collisions and $g=980 \mathrm{~cm} / \mathrm{s}^{2}$.

Then the O-helium abundance the Earth is determined by the equilibrium between the in-falling and down-drifting fluxes.

The in-falling O-helium flux from dark matter halo is

$$
F=\frac{n_{0}}{8 \pi} \cdot\left|\overline{V_{h}}+\overline{V_{E}}\right|,
$$

where $V_{h}$-speed of Solar System $(220 \mathrm{~km} / \mathrm{s}), V_{E}$-speed of Earth $(29.5 \mathrm{~km} / \mathrm{s})$ and $n_{0}=3 \cdot 10^{-4} S_{3}^{-1} \mathrm{~cm}^{-3}$ is the local density of O-helium dark matter. Here, for simplicity, we don't take into account velocity dispersion and distribution of particles in the incoming flux that can lead to significant effect.

At a depth $L$ below the Earth's surface, the drift timescale is $t_{d r} \sim L / V$, where $V \sim 400 S_{3} \mathrm{~cm} / \mathrm{s}$ is given by Eq. (3). It means that the change of the incoming flux, 
caused by the motion of the Earth along its orbit, should lead at the depth $L \sim$ $10^{5} \mathrm{~cm}$ to the corresponding change in the equilibrium underground concentration of $\mathrm{OHe}$ on the timescale $t_{d r} \approx 2.5 \cdot 10^{2} S_{3}^{-1} \mathrm{~s}$.

In underground detectors, $\mathrm{OHe}$ "atoms" are slowed down to thermal energies and give rise to energy transfer $\sim 2.5 \cdot 10^{-4} \mathrm{eVA} / S_{3}$, far below the threshold for direct dark matter detection. It makes this form of dark matter insensitive to the severe CDMS constraints 29. However, in $\mathrm{OHe}$ reactions with the matter of underground detectors can lead to observable effects.

The equilibrium concentration, which is established in the matter of underground detectors, is given by

$$
n_{o E}=\frac{2 \pi \cdot F}{V}=n_{o E}^{(1)}+n_{o E}^{(2)} \cdot \sin \left(\omega\left(t-t_{0}\right)\right),
$$

where $\omega=2 \pi / T, T=1 y r$ and $t_{0}$ is the phase. The averaged concentration is given by

$$
n_{o E}^{(1)}=\frac{n_{o}}{320 S_{3} A_{\text {med }}^{1 / 2}} V_{h}
$$

and the annual modulation of concentration is characterized by

$$
n_{o E}^{(2)}=\frac{n_{o}}{640 S_{3} A_{m e d}^{1 / 2}} V_{E}
$$

The rate of nuclear reactions of OHe with nuclei is proportional to the local concentration and the energy release in these reactions should lead to observable signal. There are two parts of the signal: the one determined by the constant part and annual modulation, which is concerned by the strategy of dark matter search in DAMA experiment 22 .

\subsection{Low energy bound state of O-helium with nuclei}

Our explanation $19 \mid 20$ is based on the idea that OHe, slowed down in the matter of DAMA/NaI or DAMA/LIBRA detector, can form a few $\mathrm{keV}$ bound state with nucleus, in which $\mathrm{OHe}$ is situated beyond the nucleus. Therefore the positive result of this experiment is explained by reaction

$$
A+\left({ }^{4} H e^{++} X^{--}\right) \rightarrow\left[A\left({ }^{4} H e^{++} X^{--}\right)\right]+\gamma
$$

with sodium and/or iodine. In detectors with different chemical content such level may not exist at all, or has other value of energy. The rate of reaction (7) is proportional to temperature and suppressed in cryogenic detectors, making the comparison of their results with DAMA a nontrivial task.

The approach of Refs. 19 and 20 assumes the following picture: at the distances larger, than its size, OHe is neutral and it feels only Yukawa exponential tail of nuclear attraction, due to scalar-isoscalar nuclear potential. It should be noted that scalar-isoscalar nature of He nucleus excludes its nuclear interaction due to $\pi$ or $\rho$ meson exchange, so that the main role in its nuclear interaction outside the nucleus 
plays $\sigma$ meson exchange, on which nuclear physics data are not very definite. When the distance from the surface of nucleus becomes smaller than the size of OHe, the mutual attraction of nucleus and $\mathrm{OHe}$ is changed by dipole Coulomb repulsion. Inside the nucleus strong nuclear attraction takes place. In the result the spherically symmetric potential appears, given by

$$
U=-\frac{A_{H e} A g^{2} \exp (-\mu r)}{r}+\frac{Z_{H e} Z e^{2} r_{o} \cdot F(r)}{r^{2}} .
$$

Here $A_{H e}=4, Z_{H e}=2$ are atomic weight and charge of helium, $A$ and $Z$ are respectively atomic weight and charge of nucleus, $\mu$ and $g^{2}$ are the mass and coupling of scalar-isoscalar meson - mediator of nuclear attraction, $r_{o}$ is the size of $\mathrm{OHe}$ and $F(r)$ is its electromagnetic formfactor, which strongly suppresses the strength of dipole electromagnetic interaction outside the OHe "atom".

To simplify the solution of Schrodinger equation the potential (8) was approximated in 19 by a rectangular potential, presented on Fig. 1.

Solutions of Schrodinger equation for each of the four regions, indicated on Fig. [1. are given in textbooks (see e.g. 30 ) and their sewing determines the condition, under which a low-energy OHe-nucleus bound state appears in the region III.

The energy of this bound state and its existence strongly depend on the parameters $\mu$ and $g^{2}$ of nuclear potential (8). On the Fig. 20 the region of these parameters, giving 2-6 keV energy level in OHe bound states with sodium and iodine are presented. In these calculations 19 the mass of $\mathrm{OHe}$ was taken equal to $m_{o}=1 \mathrm{TeV}$.

The rate of radiative capture of $\mathrm{OHe}$ by nuclei can be calculated with the use of the analogy with the radiative capture of neutron by proton with the account for: i) absence of M1 transition that follows from conservation of orbital momentum and ii) suppression of E1 transition in the case of OHe. Since OHe is isoscalar, isovector $\mathrm{E} 1$ transition can take place in OHe-nucleus system only due to effect of isospin nonconservation, which can be estimated by factor $f \sim 10^{-3}$, corresponding to relative mass difference of neutron and proton. In the result the rate of $\mathrm{OHe}$ radiative capture by nucleus with atomic number $A$ and charge $Z$ to the energy

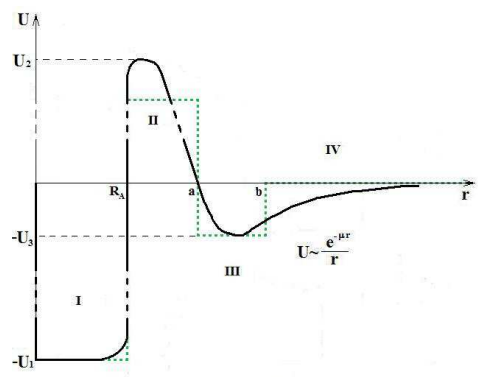

Fig. 1. The approximation of rectangular well for potential of OHe-nucleus system. 
level $E$ in the medium with temperature $T$ is given by

$$
\sigma v=\frac{f \pi \alpha}{m_{p}^{2}} \frac{3}{\sqrt{2}}\left(\frac{Z}{A}\right)^{2} \frac{T}{\sqrt{A m_{p} E}} .
$$

Formation of OHe-nucleus bound system leads to energy release of its binding energy, detected as ionization signal. In the context of our approach the existence of annual modulations of this signal in the range $2-6 \mathrm{keV}$ and absence of such effect at energies above $6 \mathrm{keV}$ means that binding energy of $\mathrm{Na}-\mathrm{OHe}$ and $\mathrm{I}-\mathrm{OHe}$ systems in DAMA experiment should not exceed $6 \mathrm{keV}$, being in the range $2-4 \mathrm{keV}$ for at least one of these elements. The amplitude of annual modulation of ionization signal (measured in counts per day per $\mathrm{kg}, \mathrm{cpd} / \mathrm{kg}$ ) is given by

$\zeta=\frac{3 \pi \alpha \cdot n_{o} N_{A} V_{E} t Q}{640 \sqrt{2} A_{m e d}^{1 / 2}\left(A_{I}+A_{N a}\right)} \frac{f}{S_{3} m_{p}^{2}}\left(\frac{Z_{i}}{A_{i}}\right)^{2} \frac{T}{\sqrt{A_{i} m_{p} E_{i}}}=4.3 \cdot 10^{10} \frac{f}{S_{3}^{2}}\left(\frac{Z_{i}}{A_{i}}\right)^{2} \frac{T}{\sqrt{A_{i} m_{p} E_{i}}}$.

Here $N_{A}$ is Avogadro number, $i$ denotes Na or I, $Q=10^{3}$ (corresponding to $1 \mathrm{~kg}$ of the matter of detector), $t=86400 \mathrm{~s}, E_{i}$ is the binding energy of $\mathrm{Na}-\mathrm{OHe}$ (I-OHe) system and $n_{0}=3 \cdot 10^{-4} S_{3}^{-1} \mathrm{~cm}^{-3}$ is the local density of O-helium dark matter. The value of $\zeta$ should be compared with the integrated over energy bins signals in DAMA/NaI and DAMA/LIBRA experiments and the result of these experiments can be reproduced e.g. for $E_{N a}=3 \mathrm{keV}$ and $E_{I}=5 \mathrm{keV}$.

At the corresponding values of $\mu$ and $g^{2}$ energy of OHe binding with other nuclei can strongly differ from 2-6 keV. In particular, energy release at the formation of

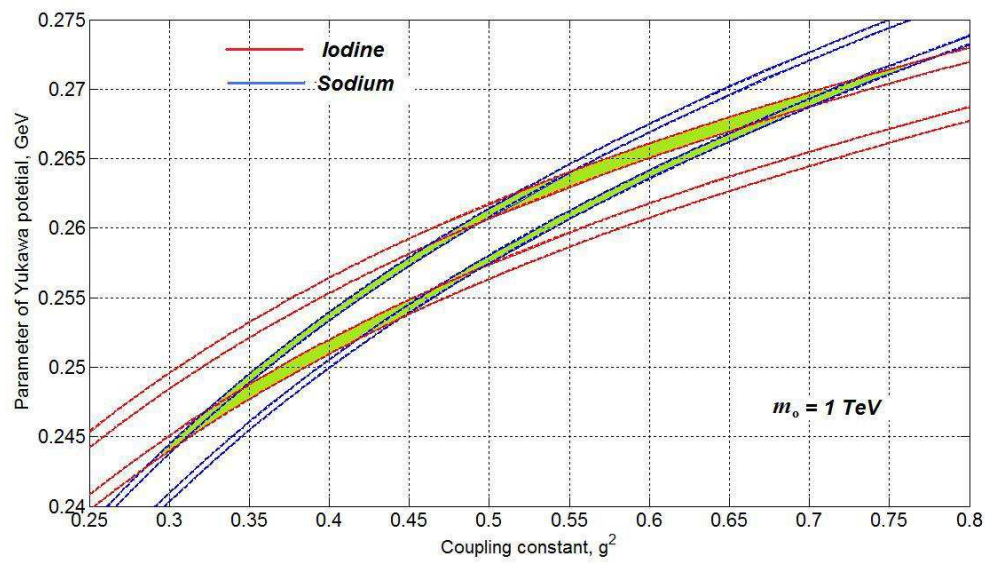

Fig. 2. The region of parameters $\mu$ and $g^{2}$, for which $\mathrm{Na}$ and I have a level in the interval 2-6 keV. For each nucleus two narrow strips determine the region of parameters, at which the bound system of this element with OHe has a level in 2-6 keV energy range. The outer line of strip corresponds to the level of $6 \mathrm{keV}$ and the internal line to the level of $2 \mathrm{keV}$. The region of intersection of strips correspond to existence of $2-6 \mathrm{keV}$ levels in both OHe-Na and OHe-I systems, while the piece of strip between strips of other nucleus corresponds to the case, when OHe bound state with this nucleus has 2-6 keV level, while the binding energy of OHe with the other nuclei is less than $2 \mathrm{keV}$ by absolute value. 
OHe bound state with thallium can be larger than $6 \mathrm{keV}$. However, for the cross section of radiative capture of thallium by OHe, given by Eq. (9) and taking into account that thallium content in DAMA detector is 3 orders of magnitude smaller, than NaI, such signal is below the experimental errors.

It should be noted that the results of DAMA experiment exhibit also absence of annual modulations at the energy of $\mathrm{MeV}$-tens $\mathrm{MeV}$. Energy release in this range should take place, if OHe-nucleus system comes to the deep level inside the nucleus (in the region I of Fig. (1). This transition implies tunneling through dipole Coulomb barrier and is suppressed below the experimental limits. The actual rate of these transitions is under our current study.

Since OHe capture rate is proportional to the temperature, it is suppressed in cryogenic detectors by a factor of order $10^{-4}$. The predicted effects of OHe radiative capture in different cryogenic detectors at $T=10 \mathrm{mK}, f=10^{-3}$ and $S_{3}=1$ are given in Table 1

Table 1. Effects of OHe in cryogenic detectors.

\begin{tabular}{lccc}
\hline Content & $\begin{array}{c}\text { Binding energy } \\
(\mathrm{keV})\end{array}$ & $\begin{array}{c}\text { Capture rate } \\
\left(10^{-33} \mathrm{~cm}^{3} / \mathrm{s}\right)\end{array}$ & $\begin{array}{c}\text { Counts per day } \\
\left(10^{-7} \mathrm{cpd} / \mathrm{kg}\right)\end{array}$ \\
\hline $\mathrm{Ge}$ & 8.6 & 4.9 & 7.2 \\
$\mathrm{Xe}$ & 6.1 & 4.4 & 3.5 \\
$\mathrm{Ar}$ & 15.6 & 4.8 & 12.8 \\
$\mathrm{C}$ & 398.5 & 2.0 & 17.8 \\
\hline
\end{tabular}

\section{Conclusions}

To conclude, the existence of heavy stable charged particles may not only be compatible with the experimental constraints but even lead to composite dark matter scenario of nuclear interacting Warmer than Cold Dark Matter. This new form of dark matter can provide explanation of excess of positron annihilation line radiation, observed by INTEGRAL in the galactic bulge. The search for stable -2 charge component of cosmic rays is challenging for PAMELA and AMS02 experiments. Decays of heavy charged constituents of composite dark matter can provide explanation for anomalies in spectra of cosmic high energy positrons and electrons, observed by PAMELA, FERMI and ATIC. In the context of our approach search for heavy stable charged quarks and leptons at LHC acquires the significance of experimental probe for components of cosmological composite dark matter.

The results of dark matter search in experiments DAMA/NaI and DAMA/LIBRA can be explained in the framework of our scenario without contradiction with negative results of other groups. Our approach contains distinct features, by which the present explanation can be distinguished from other recent approaches to this problem 31 (see also review and more references in Ref. 32).

The proposed explanation is based on the mechanism of low energy binding of 
OHe with nuclei. Within the uncertainty of nuclear physics parameters there exists a range at which $\mathrm{OHe}$ binding energy with sodium and/or iodine is in the interval $2-6 \mathrm{keV}$. Radiative capture of $\mathrm{OHe}$ to this bound state leads to the corresponding energy release observed as an ionization signal in DAMA detector.

OHe concentration in the matter of underground detectors is determined by the equilibrium between the incoming cosmic flux of $\mathrm{OHe}$ and diffusion towards the center of Earth. It is rapidly adjusted and follows the change in this flux with the relaxation time of few minutes. Therefore the rate of radiative capture of OHe should experience annual modulations reflected in annual modulations of the ionization signal from these reactions.

An inevitable consequence of the proposed explanation is appearance in the matter of DAMA/NaI or DAMA/LIBRA detector anomalous superheavy isotopes of sodium and/or iodine, having the mass roughly by $m_{o}$ larger, than ordinary isotopes of these elements.

Our results show that the ionization signal, detected by DAMA, is proportional to the temperature and should be suppressed in cryogenic detectors. Therefore test of results of DAMA/NaI and DAMA/LIBRA experiments by other experimental groups can become a very nontrivial task, especially, in view of their rejection of electromagnetic part of counting rate in the absence of nuclear recoil.

The presented approach sheds new light on the physical nature of dark matter. Specific properties of composite dark matter and its constituents are challenging for their experimental search. OHe interaction with matter is an important aspect of these studies. In this context positive result of DAMA/NaI and DAMA/LIBRA experiments may be a signature for exciting phenomena of O-helium nuclear physics.

\section{Acknowledgments}

We would like to thank Pierluigi Belli, Rita Bernabei, Jean Pierre Gazeau and Bernard Sadoulet for discussions and important comments. We express our gratitude to organizers of IWARA09 for cooperation.

\section{References}

1. M.Yu. Khlopov Cosmoparticle physics (World Scientific, Singapore, 1999).

2. M.Yu. Khlopov in Cosmion-94, Eds. M.Yu.Khlopov et al. (Editions frontieres, 1996) P. 67; M. Y. Khlopov, Bled Workshops in Physics 7 (2006) 51.

3. M. Y. Khlopov, Bled Workshops in Physics 8 (2007) 114 ; in arXiv:0711.4681, p. 114; M. Y. Khlopov and N. S. Mankoc-Borstnik, ibid, p. 195.

4. S. L. Glashow, arXiv:hep-ph/0504287

5. D. Fargion and M. Khlopov, arXiv:hep-ph/0507087

6. K.M.Belotsky et al., Gravitation and Cosmology 11 (2005) 3

7. M.Yu. Khlopov, JETP Lett. 83 (2006) 1 .

8. K. Belotsky et al., arXiv:astro-ph/0602261. K. Belotsky et al., Gravitation and Cosmology 12 (2006) 1 ; K. Belotsky et al., arXiv:0806.1067 [astro-ph].

9. M. Y. Khlopov, arXiv:astro-ph/0607048.

10. C. A. Stephan, arXiv:hep-th/0509213. 
11. D. Fargion et al., Class. Quantum Grav. 23 (2006) 7305 ; M. Y. Khlopov and C. A. Stephan, arXiv:astro-ph/0603187.

12. A. Connes Noncommutative Geometry (Academic Press, London and San Diego, 1994).

13. M. Y. Khlopov and C. Kouvaris, Phys. Rev. D 77 (2008) 065002 .

14. F. Sannino and K. Tuominen, Phys. Rev. D 71 (2005) 051901 ; D. K. Hong et al., Phys. Lett. B 597 (2004) 89 ; D. D. Dietrich et al., Phys. Rev. D 72 (2005) 055001 ; S. B. Gudnason et al., Phys. Rev. D 73 (2006) 115003 ; S. B. Gudnason et al, Phys. Rev. D 74 (2006) 095008.

15. N.S. Mankoč Borštnik, Mod. Phys. Lett. A 10 (1995) 587 ; N.S. Mankoč Borštnik, Int. J. Theor. Phys. 40 (2001) 315 ; G. Bregar, M. Breskvar, D. Lukman, N.S. Mankoč Borštnik, New J. of Phys. 10 (2008) 093002.

16. C. B. Dover et al., Phys. Rev. Lett. 42 (1979) 1117 ; S. Wolfram, Phys. Lett. B 82 (1979) 65 ; G. D. Starkman et al., Phys. Rev. D 41 (1990) 3594 ; R. Bernabei et al., Phys. Rev. Lett. 83 (1999) 4918 ; D. Javorsek et al., Phys. Rev. Lett. 87 (2001) 231804 ; S. Mitra, Phys. Rev. D 70 (2004) 103517 ; G. D. Mack et al., Phys. Rev. D 76 (2007) 043523 ; B. D. Wandelt et al., arXiv:astro-ph/0006344; P. C. McGuire and P. J. Steinhardt, arXiv:astro-ph/0105567; G. Zaharijas and G. R. Farrar, Phys. Rev. D 72 (2005) 083502 .

17. M. Y. Khlopov, arXiv:0806.3581 [astro-ph];

M. Y. Khlopov, arXiv:0801.0167] [astro-ph]; arXiv:0801.0169] [astro-ph]. M. Yu. Khlopov et al., Bled Workshops in Physics 9 (2008) 24

18. M. Y. Khlopov and C. Kouvaris, Phys. Rev. D 78 (2008) 065040

19. M. Yu. Khlopov, A. G. Mayorov, E.Yu. Soldatov, Bled Workshops in Physics 10 (2009) 79 ; arXiv:0911.5606 [astro-ph];

20. M. Yu. Khlopov, The puzzles of dark matter searches, to appear in AIP Proceedings of the 'Invisible Universe International Conference', arXiv:0911.5685 [astro-ph.CO].

21. R. Bernabei et al., Rivista Nuovo Cimento 26 (2003) 1

22. DAMA Collaboration (R. Bernabei et al.), Eur.Phys.J C56 (2008) 333 ; R. Bernabei et al., arXiv:1002.1028 [astro-ph.GA].

23. R. N. Cahn and S. L. Glashow, Science 213 (1981) 607 ; M. Pospelov, Phys. Rev. Lett. 98 (2007) 231301 ; K. Kohri and F. Takayama, Phys. Rev. D 76 (2007) 063507.

24. K. M. Belotsky, A. G. Mayorov and M.Yu.Khlopov, "Charged particles of dark matter in the cosmic rays", to appear in Proc. of the Scientific Session MIFI-2010

25. D. P. Finkbeiner and N. Weiner, Phys. Rev. D 76 (2007) 083519

26. B. J. Teegarden et al., Astrophys. J. 621 (2005) 296

27. D. McCammon et al., Nucl. Instrum. Methods A 370 (1996) 266 ; D. McCammon et al., Astrophys. J. 576 (2002) 188.

28. K. Belotsky et al., arXiv:astro-ph/0606350

29. CDMS Collaboration (D. S. Akerib et al.), Phys. Rev. Lett. 96 (2006) 011302 ; CDMS Collaboration (Z. Ahmed et al.), Phys. Rev. Lett. 102 (2009) 011301 ; The CDMS-II Collaboration (Z. Ahmed et al.), arXiv:0912.3592 [astro-ph.CO]; O. Kamaev for the CDMS Collaboration, arXiv:0910.3005 [hep-ex].

30. L. D. Landau, E. M. Lifshitz Quantum Mechanics: Non-Relativistic Theory (Fizmatlit, Moscow, 2004).

31. F. Petriello and K. M. Zurek, JHEP 0809 (2008) 047 ; R. Foot, Phys. Rev. D 78 (2008) 043529 ; J. L. Feng, J. Kumar and L. E. Strigari, arXiv:0806.3746 [hep-ph]; J. L. Feng, J. Kumar, J. Learned and L. E. Strigari, arXiv:0808.4151 [hep-ph]; E. M. Drobyshevski, arXiv:0811.0151 [astro-ph].

32. G. B. Gelmini, arXiv:0810.3733 [hep-ph]. 